\title{
The effect of thermal expansion on diffusion flame instabilities $\dagger$
}

\author{
M. MATALON ${ }^{1} \ddagger$ AND P. METZENER ${ }^{2}$ \\ ${ }^{1}$ Department of Mechanical Science and Engineering, University of Illinois at Urbana-Champaign, \\ Urbana, IL 61801, USA \\ ${ }^{2}$ Faculté des Sciences de Base, Ecole Polytechnique Fédérale de Lausanne, 1015 Lausanne, Switzerland
}

(Received 19 August 2009; revised 19 October 2009; accepted 22 October 2009)

In this paper we examine the effect of thermal expansion on the stability of a planar unstrained diffusion flame and provide a comprehensive characterization of diffusivethermal instabilities while realistically accounting for density variations. The possible patterns that are likely to be observed as a result of differential and preferential diffusion are identified for a whole range of parameters including the distinct Lewis numbers associated with the fuel and oxidizer, the initial mixture strength and the flow conditions. Although we find that thermal expansion has a marked influence on flame instability, it does not play a crucial role as it does in premixed combustion. It primarily affects the parameter regime associated with the onset of the instabilities and the growth rate of the unstable modes. Perhaps the most surprising result is that its has a different influence on the various modes of instability - a destabilizing influence on the formation of cellular structures and a stabilizing influence on the onset of oscillations.

\section{Introduction}

A well-known result in combustion that has a pronounced effect on the shape and propagation of premixed flames is the hydrodynamic or Darrieus-Landau instability, which has been discovered nearly 70 years ago in purely theoretical studies by Darrieus (1938) and Landau (1944). The instability is due to hydrodynamic effects associated with the gas expansion, or density drop across the flame, and is dominant in sufficiently large flames where the influences of diffusion are minimal. The appearance of sharp folds and creases on the surface of premixed flames in low-intensity turbulent flows and the roughened surface observed on sufficiently large centrally ignited flames are direct manifestations of the hydrodynamic instability. In contrast, the effect of thermal expansion on non-premixed flames has not been studied before. Although its role in diffusion flames may not be as pronounced as in premixed flames, its influence must nevertheless be properly assessed.

There is ample experimental evidence of intrinsic instabilities in diffusion flames. One form of instability is the breakup of a smooth flame surface into cells. The first known study is due to Gardside \& Jackson (1951) who observed that the surface of a hydrogen-air jet flame often comprises of triangular cells in the shape of a polyhedron. Later Dongworth \& Melvin (1976) found that under certain conditions the straight base of a hydrogen-oxygen diffusion flame on top of a splitter-plate burner becomes

$\dagger$ This paper is dedicated to Steve H. Davis on the occasion of his 70th birthday. As former Colleague (M. Matalon) and Postdoctoral Associate (P. Metzener) we are both indebted to Steve for his friendship and mentorship.

$\ddagger$ Email address for correspondence: matalon@illinois.edu 
cellular. Cellular structures were also observed by Chen, Mitchell \& Ronney (1992) at the base of a Wolfhard-Parker burner flame, by Lo Jacono, Papas \& Monkewitz (2003) and Lo Jacono \& Monkewitz (2007) in jet flames and by Ishizuka \& Tsuji (1981) in a counter-flow flame. A common feature of all these observations is that the instability results at sufficiently high flow rates, or at near-extinction conditions. Another feature is that cells form when at least one of the Lewis numbers, which represent the ratio of thermal-to-mass diffusivities, is sufficiently small. For example, they were observed in hydrogen-oxygen flames diluted in $\mathrm{CO}_{2}, \mathrm{~N}_{2}$ or $\mathrm{Ar}$, in which case the Lewis number associated with the fuel $L_{F} \sim 0.33-0.35$, but not when diluted in He because $L_{F}$ is near one and the Lewis number associated with the oxidizer $L_{X}$ is quite large (between 2 and 3). Similarly cells were observed in methane-air diluted in $\mathrm{SF}_{6}$, in which case $L_{F}, L_{X} \sim 0.4-0.5$, but not when diluted in $\mathrm{He}$ or $\mathrm{N}_{2}$ because the Lewis numbers are again near or larger than 1 . Finally, the propensity of diffusion flames to form cells is increased when decreasing the initial mixture strength $\phi$, defined as the ratio of the fuel-to-oxidizer mass supplied in their respective streams, normalized by their stoichiometric proportions.

Another form of instability common in diffusion flames is the spontaneous onset of oscillations. Oscillations were observed by Chan \& Tien (1979) during the combustion of condensed-phase fuels, by Ross, Sotos \& T'ien (1991) for candle flames in a microgravity environment, by Füri, Papas \& Monkewitz (2000) for jet flames, by Golovanevsky et al. (1999) for spray flames and by Ross (1994) and Schiller, Ross \& Sirignano (1996) for flame spreading over liquid beds. A common feature of all these observations is that the instability results at sufficiently high flow rates, or at nearextinction conditions, when one of the Lewis numbers is sufficiently large. Oscillations are also more likely to occur when the initial mixture strength $\phi$ is sufficiently large (Füri et al. 2000), namely for relatively rich conditions. The nature of the oscillation in each of these experiments, however, is quite different: The droplet flame exhibits radial oscillations and the jet flame expands and contracts as a whole during a cycle. For the microgravity candle flame the edge is seen to move back and forth along the hemispherical flame surface. In flame spreading the oscillations are primarily seen near the edge, decaying along the trailing diffusion flame.

The underlying hydrodynamics in the reported experiments is generally nontrivial, involving multidimensional shear and strained flows that produce non-uniform conditions over the flame surface and complicate analytical description. Stability analyses (Kim, Williams \& Ronney 1996; Cheatham \& Matalon 2000; Kukuck \& Matalon 2001; Vance, Miklavcic \& Wichman 2001; Miklavcic, Moore \& Wichman 2005; Metzener \& Matalon 2006) of diffusion flames have, therefore, predominantly used a planar flame as the basic (unperturbed) state. All these studies, however, assumed that the temperature is independent of the density, a convenient assumption that simplifies the problem by filtering out hydrodynamic disturbances. Consequently, the effect of thermal expansion on the stability of diffusion flames has not been examined. Relaxing the constant-density assumption while realistically accounting for density variations is the primary objective of this paper.

The model used in this work is based on the reaction sheet formulation proposed by Cheatham \& Matalon (2000), which exploits the limit of a large activation energy for a one-step irreversible global chemical reaction. The model consists of a nonlinear free-boundary problem for the combustion field, including the location of the reaction sheet, with conditions that permit incomplete combustion with different degrees of reactant consumption. It is not restricted to a particular geometry; it spans the entire spectrum, from complete combustion down to extinction, and allows for non-unity and distinct Lewis numbers for the fuel and oxidizer. It is therefore appropriate for analyses 
of multi-dimensional and time-dependent problems. The theory, however, is restricted to what has been termed by Liñán (1974) the 'diffusion-flame' or 'near-equilibrium' regime, excluding the 'premixed-flame' regime associated with relatively large leakage of one of the two reactants. For a constant-density flow, the flame sheet model yields an explicit dispersion relation for the growth of arbitrary disturbances in terms of the flow conditions, the Lewis numbers and the mixture strength (Cheatham \& Matalon 2000; Kukuck \& Matalon 2001). Due to its transcendental nature, finding roots for the growth rate in the complex plane requires considerable numerical computations, which led Metzener \& Matalon (2006) to re-examine the linearized stability eigenvalue problem numerically. The numerical and analytical results yielded a comprehensive characterization of the instabilities, the critical conditions for their onset and the patterns that are likely to be observed beyond the instability threshold. For a variabledensity flow the problem is far more complicated; it consists of equations with variable coefficients that cannot be addressed analytically and their discretization leads to a generalized eigenvalue problem with large matrices that are not necessarily invertible. This requires a careful examination of the spectrum in the complex plane, separating the continuous part from the discrete eigenvalues and focusing with sufficient accuracy on the fastest growing mode in order to extract reliable stability results. Moreover, the problem involves a large number of parameters that include the Lewis numbers associated with fuel and oxidizer, the initial mixture fraction, the temperature and density differences between the reactants at their supply boundaries, the hot-to-cold density ratio and the flow conditions.

\section{Formulation}

The configuration adopted in this work is the chambered diffusion flame, in which one of the reactants is fed from the bottom of the chamber at a constant rate and the other diffuses uniformly from the top of the chamber against the flow of products. The uniform conditions in the horizontal cross-section at the top of the chamber are achieved by introducing the reactant through an array of closely laid-out hypodermic needles, spaced equally to allow the hot combustion products to escape between the needles. The inhomogeneity near the tip of the needles is limited to a thin layer comparable to the needle spacing, so that uniform reactant concentration is established at the virtual location $x=0$, just below the needles, as shown in the schematic of figure 1(a). Under such conditions a steady planar diffusion flame could be sustained in the chamber. This idealized configuration has been experimentally verified only recently by Lo Jacono et al. (2005). Slight imperfections in the original experiment have been greatly circumvented in the latest design by Robert \& Monkewitz (2009), which permit better control of the magnitude and direction of the bulk flow. Figure 1(b) shows a side-view photograph of the planar unstrained flame. Measured residual strain along the flame front was found to be extremely small and is associated with non-uniformities resulting from heat loss to the chamber walls that could be significantly minimized by heat-loss management. The realization of an unstrained planar diffusion flame provides the opportunity to investigate fundamental questions related to flame instabilities and allows experimental validation of the results of theoretical and numerical models. Results on the formation of cells and planar pulsations have been already reported by Robert (2008). The discussion presented below refers to a set-up in which fuel is supplied in the upcoming stream, as shown in the figure. However, the results can be easily applied to the reverse configuration in which fuel diffuses against a stream of oxidizer, by simply interchanging the role of fuel and oxidizer. 


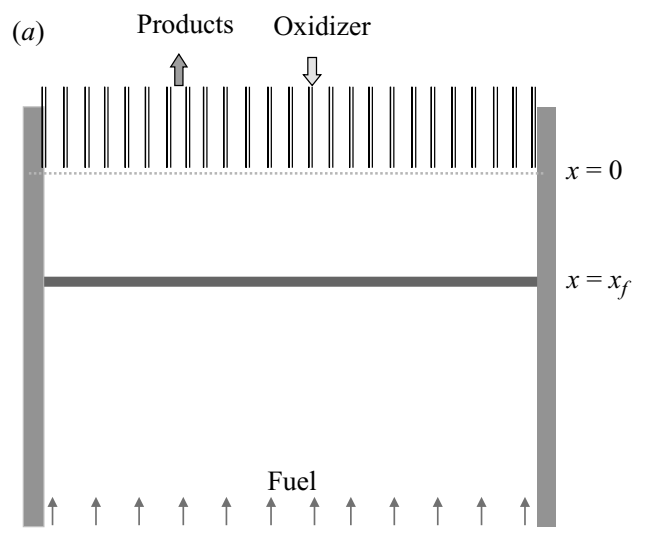

(b)

FiguRE 1. (a) Schematic of the chambered diffusion flame; fuel is supplied in the upcoming stream fed uniformly at the bottom of the chamber and oxidizer diffuses uniformly against the stream from the top boundary $x=0$. (b) Side-view photograph of a planar unstrained diffusion flame; courtesy of E. Robert and P. Monkewitz.

The chemical reaction is modelled by a global one-step irreversible reaction of the form

$$
v_{F} \text { Fuel }+v_{X} \text { Oxidizer } \rightarrow\left(v_{F}+v_{X}\right) \text { Products, }
$$

where the subscripts $F$ and $X$ denote fuel and oxidizer, respectively, and $v_{F}, v_{X}$ are the stoichiometric coefficients. The reaction rate is assumed to obey an Arrhenius law with a pre-exponential factor $\mathscr{B}$ and overall activation energy $E$. Let the fuel mass fraction in the feed stream be $Y_{-\infty}$ and the oxidizer mass fraction at the top boundary be $X_{0}$, the initial mixture strength representing the ratio of the fuel-to-oxidizer mass supplied at the respective boundaries and normalized by their stoichiometric proportions is given by

$$
\phi=\frac{Y_{-\infty} / X_{0}}{v_{F} W_{F} / v_{X} W_{X}},
$$

where $W_{F}, W_{X}$ are the molecular weights. Both reactants are diluted with inert gas that together with the combustion products constitute the abundance of the mixture and determine its properties: the thermal diffusivity $\mathscr{D}_{t h}$, the viscosity $\mu$, the specific heat at constant pressure $c_{p}$ and the average molecular weight $\bar{W}$, all assumed constants. Diffusion of each of the two reactants obeys Fick's law of binary diffusion with mass diffusivities $\mathscr{D}_{F}, \mathscr{D}_{X}$, assumed to vary with temperature such that $\rho_{\mathscr{D}_{F}}$ and $\rho \mathscr{D}_{X}$ remain constant.

\subsection{The reaction sheet model}

The formulation adopted in this work is based on the general asymptotic theory developed in Cheatham \& Matalon (2000), which exploits the limit of large activation energy. The parameter $\epsilon=c_{p} R^{o} \widetilde{T}_{s}^{2} / q E$ is assumed small, as is typical for chemical reactions encountered in combustion. Here $q=Q Y_{-\infty} / v_{F} W_{F}$ is the heat released per unit mass of fuel supplied with $Q$ the total heat release, $R^{o}$ is the gas constant and $\widetilde{T}_{s}$ the stoichiometric temperature associated with complete combustion (determined below). In this limit the chemical activity is confined to a sheet, $F(\boldsymbol{x}, t)=0$, that divides the combustion field into two regions: the fuel region corresponding to $F<0$ and the oxidizer region corresponding to $F>0$. The thin reaction zone centred at $F=0$ remains quasi-planar and quasi-steady and its structure, resolved on the $O(\epsilon)$ scale, imposes conditions across, and at the reaction sheet that depend on the overall heat 
release, the intensity of the chemical reaction and the degree of reactant consumption. Unlike the fast chemistry limit, where the fuel and oxidizer are completely consumed along the stoichiometric surface as envisaged by Burke \& Schumann (1928), the present theory allows for reactant leakage through the sheet and is therefore applicable to conditions that extend from complete combustion down to extinction.

Due to the low velocities, the combustion process is nearly isobaric implying that pressure variations from the (constant) ambient pressure $P_{0}$ are small. The equation of state then implies that the density of the mixture is inversely proportional to the temperature. In the following the ambient pressure $P_{0}$ is taken as a unit of pressure and $q / c_{p}$ as a unit of temperature; the characteristic density is then $P_{0} \bar{W} c_{p} / q R^{o}$. The uniform velocity $U$ far upstream is chosen as a unit speed, the diffusion length $l_{D}=\mathscr{D}_{t h} / U$ as a unit length and $\mathscr{D}_{t h} / U^{2}$ as a unit time. The mass fractions of fuel and oxidizer are normalized with respect to $Y_{-\infty}$ and $\phi X_{0}$, respectively. The governing equations on either side of the reaction sheet (in dimensionless form) are

$$
\begin{gathered}
\frac{\mathrm{D} \rho}{\mathrm{D} t}+\rho \nabla \cdot \boldsymbol{v}=0 \\
\rho \frac{\mathrm{D} \boldsymbol{v}}{\mathrm{D} t}=-\nabla p+\operatorname{Pr}\left(\nabla^{2} \boldsymbol{v}+\frac{1}{3} \nabla(\nabla \cdot v)\right), \\
\rho \frac{\mathrm{D} T}{\mathrm{D} t}-\nabla^{2} T=0 \\
\rho \frac{\mathrm{D} Y}{\mathrm{D} t}-L_{F}^{-1} \nabla^{2} Y=0 \\
\rho \frac{\mathrm{D} X}{\mathrm{D} t}-L_{X}^{-1} \nabla^{2} X=0 \\
\rho T=1 .
\end{gathered}
$$

where $\boldsymbol{v}, p$ are the velocity and pressure fields, $\rho, T$ the density and temperature and $Y, X$ the mass fractions of the fuel and oxidizer, respectively. The operator $\mathrm{D} / \mathrm{D} t \equiv \partial / \partial t+\boldsymbol{v} \cdot \nabla$ is the convective derivative. The parameters appearing in these equations are the Prandtl number $\operatorname{Pr}=\mu c_{p} / \lambda$, where $\lambda$ is the (constant) thermal conductivity of the mixture, and the Lewis numbers $L_{F}=\mathscr{D}_{F} / \mathscr{D}_{t h}$ and $L_{X}=\mathscr{D}_{X} / \mathscr{D}_{t h}$. The conditions at the reaction sheet are

$$
\begin{gathered}
\llbracket \boldsymbol{v} \cdot \boldsymbol{n} \rrbracket=\llbracket \boldsymbol{n} \times(\boldsymbol{v} \times \boldsymbol{n}) \rrbracket=0, \\
\llbracket \frac{\partial}{\partial n}(\boldsymbol{n} \times(\boldsymbol{v} \times \boldsymbol{n})) \rrbracket=\llbracket \nabla_{\perp}(\boldsymbol{v} \cdot \boldsymbol{n}) \rrbracket, \\
\llbracket p \rrbracket=\frac{4}{3} \operatorname{Pr} \llbracket \frac{\partial}{\partial n}(\boldsymbol{v} \cdot \boldsymbol{n}) \rrbracket+\frac{1}{3} \operatorname{Pr} \llbracket \nabla \cdot(\boldsymbol{n} \times(\boldsymbol{v} \times \boldsymbol{n})) \rrbracket, \\
\llbracket T \rrbracket=\llbracket Y \rrbracket=\llbracket X \rrbracket=0, \\
\llbracket \frac{\partial T}{\partial n}+L_{F}^{-1} \frac{\partial Y}{\partial n} \rrbracket=\llbracket \frac{\partial T}{\partial n}+L_{X}^{-1} \frac{\partial X}{\partial n} \rrbracket=0, \\
\left.Y\right|_{F=0^{+}}=\epsilon L_{F} S_{F}(\gamma, \delta),\left.\quad X\right|_{F=0^{-}}=\epsilon L_{X} S_{X}(\gamma, \delta),
\end{gathered}
$$

where the operator $\llbracket \cdot \rrbracket$ denotes the jump in the quantity across the sheet, i.e. the difference between the values at $F=0^{+}$and $F=0^{-}$. The vector $\boldsymbol{n}=\nabla F /|\nabla F|$ is a unit normal to the sheet, the differentiation $\partial / \partial n$ is along the normal and $\nabla_{\perp}$ denotes the surface gradient. We note parenthetically that there was a typographical error in Cheatham \& Matalon (2000) in the jump of the normal derivative of the mass flux across the sheet (page 129 of the referred paper). Since the velocity is expressed relative 
to the sheet, $V_{f}=-F_{t} /|\nabla F|$ must have been subtracted from the normal velocity on the left-hand side of the equation. This condition, however, is merely a restatement of the continuity equation and is in fact not needed in the formulation. The interfacial conditions (2.7)-(2.12) are sufficient for the determination of the combustion field across the sheet, as well as the shape and position of the sheet.

The reactant leakage (2.12) is expressed in terms of the leakage functions $S_{F}$ and $S_{X}$ and depend on the mixture strength in the reaction zone and on the intensity of the chemical reaction. Note that the magnitude of the leakage is relatively small, of order $\epsilon$. The auxiliary variables $\gamma$ and $\delta$ are determined from

$$
\gamma=\frac{\partial T /\left.\partial n\right|_{F=0^{+}}+\partial T /\left.\partial n\right|_{F=0^{-}}}{\llbracket \partial T / \partial n \rrbracket}, \quad \delta=\frac{4 L_{X} L_{F}}{\llbracket \partial T / \partial n \rrbracket^{2}} \mathbb{D e}^{h_{f}(\gamma, \delta)} .
$$

The variable $\gamma$ measures the excess of heat conducted to one side of the reaction sheet; it depends on the temperature profiles for complete combustion (i.e. the solution corresponding to $\epsilon=0$ ) and is, therefore, a function of the system parameters. It is restricted to $-1<\gamma<1$ corresponding to the 'diffusion-flame' regime as discussed in the introduction. The variable $\delta$ depends implicitly on the available enthalpy at the reaction sheet

$$
h_{f}=\frac{1}{2}(1-\gamma) h_{F}+\frac{1}{2}(1+\gamma) h_{X},
$$

where $h_{F}$ and $h_{X}$ are defined from the relations

$$
T+\left.L_{F}^{-1} Y\right|_{x=x_{f}^{+}}=T_{s}+\epsilon h_{F}, \quad T+\left.L_{X}^{-1} X\right|_{x=x_{f}^{-}}=T_{s}+\epsilon h_{X}
$$

and $T_{s}$ is the stoichiometric temperature, and on the Damköhler number

$$
\mathbb{D}=\frac{\mathscr{D}_{t h}}{U^{2}} \frac{v_{X} c_{p} \bar{W} Y_{-\infty}}{q R^{o} W_{F}}\left(\frac{R^{o} \widetilde{T}_{s}}{E}\right)^{3} \mathscr{B} P_{0} \mathrm{e}^{-E / R^{o} \widetilde{T}_{s}} .
$$

It measures the local intensity of the chemical reaction and varies along the sheet.

The notation adopted in this paper is such that, when the same symbol is used, a on top of the symbol identifies the dimensional or unscaled quantity. Thus $T_{s}=c_{p} \widetilde{T}_{s} / q$ and, similarly, $T_{-\infty}=c_{p} \widetilde{T}_{-\infty} / q$ and $\Delta T=c_{p}\left(\widetilde{T}_{0}-\widetilde{T}_{-\infty}\right) / q$ which appear below.

The leakage functions $S_{F}$ and $S_{X}$ are obtained as matching conditions when resolving the internal structure of the reaction zone. Numerical integration of the appropriate equations in this zone shows that for a given $\gamma$ two distinct solutions exist for $\delta>\delta_{c}$ and none for $\delta<\delta_{c}$. There are, therefore, two branches - the upper and the lower branches where $S_{F}$ and $S_{X}$ are both either increasing functions of $\delta$, or decreasing functions of $\delta$ (see figure 2). The functions $S_{1}$ and $S_{2}$ in the figure correspond respectively to the fuel and oxidizer leakage when $\gamma>0$, and to the oxidizer and fuel leakage when $\gamma<0$, namely

$$
S_{F}=\left\{\begin{array}{l}
S_{1} \quad 0 \leqslant \gamma<1 \\
S_{2}-1<\gamma \leqslant 0
\end{array} \quad S_{X}= \begin{cases}S_{2} & 0 \leqslant \gamma<1 \\
S_{1} & -1<\gamma \leqslant 0\end{cases}\right.
$$

Both reactants leak equally from the reaction zone to opposite sides when $\gamma=0$, but for $\gamma$ positive/negative there is excess fuel/oxidizer that remains unburned. The lower branches tend to the zero as $\delta \rightarrow \infty$, a state corresponding to the Burke-Schumann solution of complete combustion $\left(S_{F}=S_{X}=0\right)$. The upper branches tend to a state that is associated with $O(1)$ reactant leakage and is of no interest in this study. 
(a)

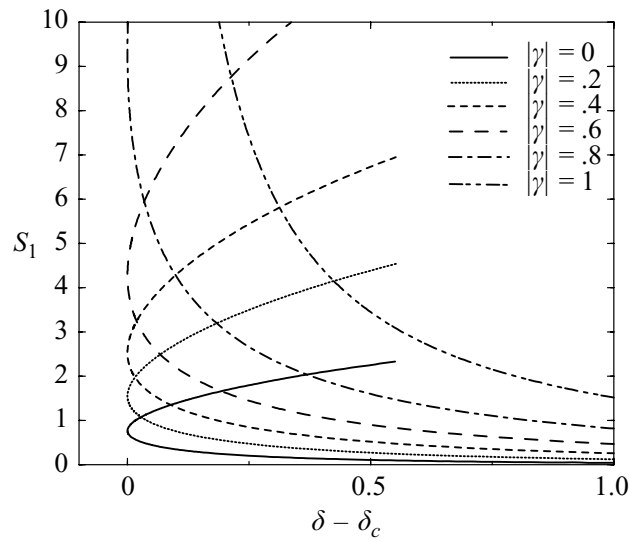

(b)

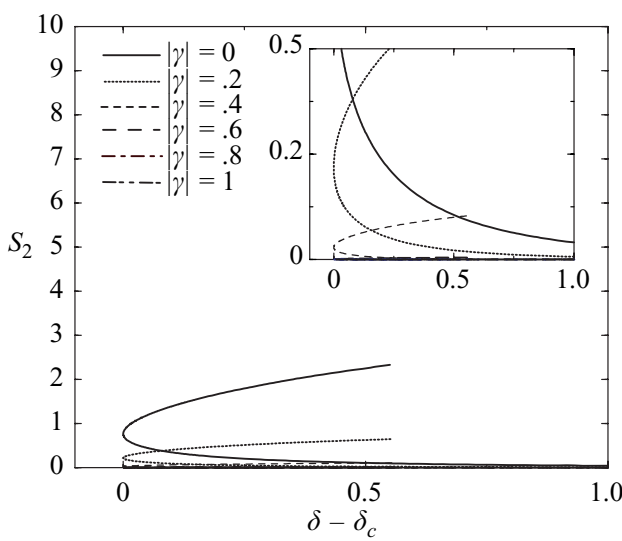

FIGURE 2. The leakage functions $S_{1}$ and $S_{2}$ as a function of $\delta-\delta_{c}$ for several values of $\gamma$,

The minimum value $\delta_{\mathrm{c}}$ below which no solution exist depends only on $\gamma$ and can be approximated by

$$
\delta_{\mathrm{c}}=\left\{1-|\gamma|-(1-|\gamma|)^{2}+0.26(1-|\gamma|)^{3}+0.055(1-|\gamma|)^{4}\right\} \mathrm{e}
$$

as shown by Liñán (1974). Since the dependence of $\delta$ on $\mathbb{D}$ is generally nonlinear, as is evident from its definition (2.13), $\delta_{c}$ does not necessarily correspond to the smallest possible value of $\mathbb{D}$. The Damköhler number, which represents the ratio of the residence time to the chemical reaction time, is controlled by the system parameters such as the ambient pressure or the flow rate (see (2.14)). The lowest possible value $\mathbb{D}=\mathbb{D}_{\text {ext }}$ denotes, therefore, conditions below which burning can no longer be sustained, or extinction conditions. The adopted notation clearly distinguishes between the two states by using different subscripts, the subscript ' $c$ ' for the critical state corresponding to $\delta_{c}$ and the subscript 'ext' corresponding to physical state associated with flame extinction.

In order to have direct access to the leakage functions and their derivatives, without the necessity to repeatedly integrate numerically the structure equations, Cheatham \& Matalon (2000) provided the following formulae

$$
\begin{aligned}
& S_{1}= \begin{cases}\delta^{-1 / 3}\left\{q_{0}+q_{1}\left(\delta-\delta_{\mathrm{c}}\right)^{q_{2}}\right\}, & \text { upper branch } \\
a_{0} \delta^{-4 / 3} \exp \left\{-a_{1}\left(\delta-\delta_{\mathrm{c}}\right)^{a_{2}}\right\}, & \text { lower branch }\end{cases} \\
& S_{2}= \begin{cases}\delta^{-1 / 3}\left\{r_{0}+r_{1}\left(\delta-\delta_{\mathrm{c}}\right)^{r_{2}}\right\}, & \text { upper branch } \\
b_{0} \delta^{-4 / 3} \exp \left\{-b_{1}\left(\delta-\delta_{\mathrm{c}}\right)^{b_{2}}\right\}, & \text { lower branch }\end{cases}
\end{aligned}
$$

which were shown to represent the numerical results presented in figure 2 with sufficient accuracy. The coefficients $a_{k}, b_{k}, q_{k}, r_{k}$ for $k=0,1,2$ depend on $|\gamma|$ and can be found in the referred paper.

The mathematical problem consists of solving equations (2.1)-(2.5) for the seven variables $\{T, Y, X, p, \boldsymbol{v}\}$ on either side of the reaction sheet, subject to (2.7)-(2.12) and appropriate boundary and initial conditions. The density $\rho$ is then obtained from the equation of state (2.6). This nonlinear free-boundary problem is a tenth-order system with eleven interfacial conditions, one of which determines the instantaneous shape of the interface $F(\boldsymbol{x}, t)=0$. 


\subsection{Simplification for two-dimensional flow}

For the linear stability discussion it is sufficient to consider two-dimensional disturbances because results for three-dimensional disturbances can then be deduced in a straightforward way. In two-dimensional the problem can be reformulated in a way that simplifies the numerical integration. Let $x, y$ denote the axial and transverse coordinates and $u, v$ the axial and transverse velocity components, respectively. Equation (2.4) combined with (2.2) and (2.6) yields $\nabla \cdot(v-\nabla T)=0$, which can be satisfied automatically if a stream-like function $\psi(x, y, t)$ is introduced such that

$$
u=\frac{\partial T}{\partial x}+\frac{\partial \psi}{\partial y}, \quad v=\frac{\partial T}{\partial y}-\frac{\partial \psi}{\partial x} .
$$

Then

$$
\nabla^{2} \psi=-\varpi .
$$

where $\varpi=\partial v / \partial x-\partial u / \partial y$ is the vorticity in the direction normal to the plane of the flow. The vorticity equation obtained as usual by taking the curl of the momentum equation, is

$$
\begin{gathered}
\rho \frac{\mathrm{D} \varpi}{\mathrm{D} t}=\operatorname{Pr} \nabla^{2} \varpi+J, \\
J=\left(\frac{\partial \rho}{\partial y} \frac{\partial u}{\partial t}-\frac{\partial \rho}{\partial x} \frac{\partial v}{\partial t}\right)+\left(\frac{\partial \rho u}{\partial y} \frac{\partial u}{\partial x}-\frac{\partial \rho u}{\partial x} \frac{\partial v}{\partial x}\right)+\left(\frac{\partial \rho v}{\partial y} \frac{\partial u}{\partial y}-\frac{\partial \rho v}{\partial x} \frac{\partial v}{\partial y}\right) .
\end{gathered}
$$

The fluid dynamical equations now reduce to solving equations (2.18)-(2.19) for $\varpi$ and $\psi$, after which the velocities can be obtained from (2.17) and the pressure from the momentum equation

$$
\rho \frac{\mathrm{D} \boldsymbol{v}}{\mathrm{D} t}=-\nabla P+\operatorname{Pr} \nabla^{2} \boldsymbol{v},
$$

where $P=p-(1 / 3) \operatorname{Pr} \nabla \cdot v$ is the reduced pressure. Conditions (2.7)-(2.9) associated with the pressure and velocities across the reaction sheet simplify to

$$
\begin{gathered}
\llbracket u \rrbracket=\llbracket v \rrbracket=0, \\
\llbracket \varpi \rrbracket=\llbracket \partial \varpi / \partial n \rrbracket=0, \\
\llbracket P \rrbracket=\operatorname{Pr} \llbracket \boldsymbol{n} \cdot \partial \boldsymbol{v} / \partial n \rrbracket .
\end{gathered}
$$

The vorticity conditions (2.22) result from integrating (2.19) across the sheet; the first of these relations is also consistent with (2.8). The condition for the pressure results from (2.9). Finally, it is convenient to use the explicit representation $x=x_{f}(y, t)$ for the reaction sheet.

\subsection{Boundary conditions for the chambered flame}

The following boundary conditions will be imposed for the chambered flame configuration. Far upstream,

$$
T=T_{-\infty}, Y=1, X=0, u=1, v=0 \quad \text { as } x \rightarrow-\infty .
$$

It is convenient to introduce the prescribed mass flux far upstream, $M \equiv \rho u$ which, in view of the equation of state satisfies $M=1 / T_{-\infty}$. If the outflow at the exit of the chamber remains uniform, then

$$
u=1+M \Delta T, \quad \partial v / \partial x=0, \quad \text { at } x=0 .
$$

The remaining conditions are

$$
T=T_{-\infty}+\Delta T, \quad Y=0, \quad X=\phi^{-1} \quad \text { at } x=0,
$$

where $\Delta T$ is the temperature differential across the chamber. 


\section{The planar diffusion flame}

The governing equations possess a steady one-dimensional solution, corresponding to a planar flame with the reaction sheet located at $x_{f}=\eta$. Mass conservation implies that across the entire chamber

$$
\rho u=M, \quad v=0 .
$$

Correct to $O\left(\epsilon^{2}\right)$, the temperature and mass fraction profiles are

$$
\begin{aligned}
& T(x)= \begin{cases}T_{-\infty}+1+(\Delta T-1) \mathrm{e}^{M x}-\epsilon L_{F} S_{F} \frac{1-\mathrm{e}^{M x}}{1-\mathrm{e}^{M L_{F} \eta}}, & x>\eta \\
T_{-\infty}+\mathrm{e}^{M(x-\eta)}+(\Delta T-1) \mathrm{e}^{M x}-\epsilon L_{F} S_{F} \frac{1-\mathrm{e}^{M \eta}}{1-\mathrm{e}^{M L_{F} \eta}} \mathrm{e}^{M(x-\eta)}, & x<\eta\end{cases} \\
& Y(x)= \begin{cases}\epsilon L_{F} S_{F} \frac{1-\mathrm{e}^{M L_{F} x}}{1-\mathrm{e}^{M L_{F} \eta},} & x>\eta \\
1-\left(1-\epsilon L_{F} S_{F}\right) \mathrm{e}^{M L_{F}(x-\eta)}, & x<\eta\end{cases} \\
& X(x)= \begin{cases}\phi^{-1}-\left(\phi^{-1}-\epsilon L_{X} S_{X}\right) \frac{1-\mathrm{e}^{M L_{X} x}}{1-\mathrm{e}^{M L_{X} \eta}}, & x>\eta \\
\epsilon L_{X} S_{X} \mathrm{e}^{M L_{X}(x-\eta)}, & x<\eta .\end{cases}
\end{aligned}
$$

Exact expressions for $u=M T$ and $\rho=T^{-1}$ are readily available. As for the pressure, it is sufficient for the following discussion to specify the pressure gradient which can be directly computed from

$$
\frac{\mathrm{d} P}{\mathrm{~d} x}=M^{2}(P r-1) \frac{\mathrm{d} T}{\mathrm{~d} x}
$$

We note that the apparent difference between the solution presented here and that in Cheatham \& Matalon (2000) is due to the location of the reaction sheet, which is taken here as $\eta$ correct to $O(\epsilon)$ instead of the leading order term $\eta_{0}$. Since the difference is $O(\epsilon)$, it is easy to verify using a Taylor expansion that the two versions are equivalent.

The location of the reaction sheet $\eta=\eta_{0}+\epsilon \eta_{1}+O\left(\epsilon^{2}\right)$ is obtained as part of the solution after satisfying all the boundary and jump conditions. We find that

$$
\eta_{0}=-\frac{1}{M L_{X}} \ln \left(1+\phi^{-1}\right), \quad \eta_{1}=\frac{1}{M}\left(S_{X}-\frac{L_{F}}{L_{X}} \frac{1-\mathrm{e}^{M L_{X} \eta_{0}}}{1-\mathrm{e}^{M L_{F} \eta_{0}}} S_{F}\right) .
$$

Note that the location of the sheet depends primarily on the ability of the oxidizer to diffuse against the stream; the weak dependence on $L_{F}$ is due to the residual fuel resulting from incomplete combustion. The flame temperature, defined as the temperature at $x=\eta$, is given by $T_{f}=T_{s}+\epsilon T_{f_{1}}+O\left(\epsilon^{2}\right)$ where

$$
T_{s}=1+T_{-\infty}-(1-\Delta T) \mathrm{e}^{M \eta_{0}}, \quad T_{f_{1}}=-M(1-\Delta T) \eta_{1} \mathrm{e}^{M \eta_{0}}-\frac{1-\mathrm{e}^{M \eta_{0}}}{1-\mathrm{e}^{M L_{F} \eta_{0}}} L_{F} S_{F}
$$

and is shown schematically in figure 3 as a function of the Damköhler number. In the fast chemistry or Burke-Schumann limit, $\mathbb{D} \rightarrow \infty$, the flame temperature approaches the stoichiometric temperature $T_{s}$. The drop in temperature $T_{f_{1}}$ is solely due to incomplete combustion (in practical situations the temperature differential across the layer is relatively small so that $\Delta T<1$ ).

The parameter

$$
\gamma=-1+2(1-\Delta T)(1+1 / \phi)^{-1 / L_{X}}
$$

depends on the initial strength $\phi$ as well as on $\Delta T$ and $L_{X}$. When $L_{X}=1$ and $\Delta T=0$ we find that $\gamma=(1-\phi) /(1+\phi)$, which is negative/positive for $\phi$ less/bigger than 


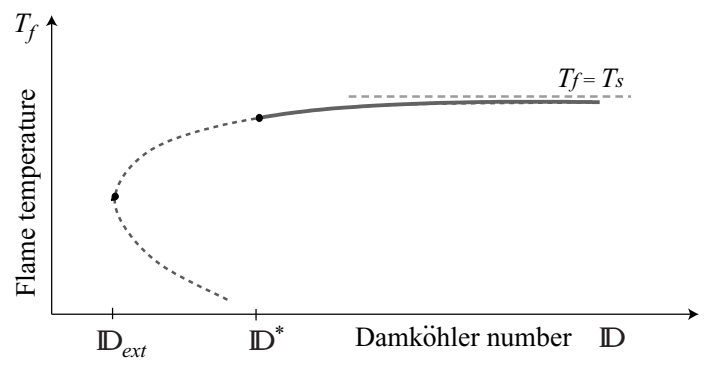

FIGURE 3. The dependence of the flame temperature of a planar diffusion flame on the Damköhler number.

one. It is, therefore, a measure of the mixture strength at the reaction sheet, which is negative/positive for lean/rich conditions. In general, the mixture strength at the reaction sheet depends also on the preferential rate at which the oxidizer diffuses to the sheet and on the additional heat available at the oxidizer supply boundary.

The available enthalpy at the reaction sheet, $h_{f}=T_{f_{1}}+(1 / 2)(1-\gamma) S_{F}+$ $(1 / 2)(1+\gamma) S_{X}$, can be expressed solely in terms of $S_{F}$ namely

$$
h_{f}=\left\{\frac{1-\gamma}{2} \frac{\left(1-\mathrm{e}^{M L_{F} \eta_{0}}\right) L_{F}^{-1}-\left(1-\mathrm{e}^{M \eta_{0}}\right)}{1-\mathrm{e}^{M L_{F} \eta_{0}}}+\frac{1+\gamma}{2} \frac{\left(1-\mathrm{e}^{M L_{X} \eta_{0}}\right) L_{X}^{-1}-\left(1-\mathrm{e}^{M \eta_{0}}\right)}{1-\mathrm{e}^{M L_{F} \eta_{0}}}\right\} L_{F} S_{F} .
$$

For complete combustion and for unity Lewis numbers $h_{f}=0$ and $\delta$ is directly proportional to the Damköhler number $\mathbb{D}$. Otherwise, there is excess/deficiency in the available enthalpy, which results from differential and preferential diffusion, i.e. non-unity and non-equal Lewis numbers. The relation

$$
\delta=\frac{4 L_{F} L_{X}}{M^{2}} \mathbb{D} \mathrm{e}^{h_{f}(\delta)}
$$

is then an implicit relation that serves to determine the auxiliary function $\delta$ for a given Damköhler number or, equivalently $\mathbb{D}$ as a function of $\delta$. The solution in terms of $\mathbb{D}$ is therefore multi-valued (since it is multi-valued in terms of $\delta$ ) with a turning point at the lowest possible value of the Damköhler number, $\mathbb{D}=\mathbb{D}_{\text {ext }}$; see, for example, the dependence of the flame temperature on $\mathbb{D}$ sketched in figure 3 . We note parenthetically that the response curve in the figure is only the upper portion of an S-response curve that characterizes diffusion flames; the lower part not shown is associated with frozen combustion and flame ignition.

It is convenient to introduce the density ratio

$$
r \equiv \rho_{-\infty} / \rho_{s}=T_{s} / T_{-\infty}
$$

which characterizes the effect of thermal expansion when both reactants are supplied at the same temperature. Heating the oxidizer introduces, in addition, a density contrast across the entire layer characterized by the parameter $\Delta T$. We note that the constant-density model used in all previous studies is not a straightforward limit process expansion of the governing equations; it is an ad-hoc model that results when replacing the equation of state (2.6) with $\rho=1$. Indeed, the constant density model can be systematically recovered if one assumes that the heat of combustion is small, but since the temperature here has been scaled with respect to the heat release, recovering this limit within the present formulation is not obvious. There are two main differences when comparing the solution (3.1)-(3.4) with that obtained using the constant-density model: the position of the flame is reduced by the factor 
$M$, and the ratio $\delta / \mathbb{D}$ is reduced by the factor $M^{2}$. All the other quantities such as $\gamma, T_{s}, T_{f}$ and $h_{f}$ are not affected since the product $M \eta$ remains constant. Apart from the multiplicative factor $M^{2}$, the dependence of $\mathbb{D}$ on $\delta$ and the dependence of $T_{f}$ on $\mathbb{D}$ are similar to those shown in figures 3 and 4 of Metzener \& Matalon (2006).

\section{Linear stability problem}

The linear stability of the planar flame is analysed by introducing small disturbances to the basic state (3.1)-(3.6), which will be identified below by the subscript $b$. Let

$$
\begin{aligned}
x_{f} & =\eta_{b}(\epsilon)+\epsilon A \exp (\omega t+\mathrm{i} \sigma y), \\
T(x, y, t) & =T_{b}(x ; \epsilon)+\epsilon A \hat{T}(x) \exp (\omega t+\mathrm{i} \sigma y), \\
Y(x, y, t) & =Y_{b}(x ; \epsilon)+\epsilon A \hat{Y}(x) \exp (\omega t+\mathrm{i} \sigma y), \\
X(x, y, t) & =X_{b}(x ; \epsilon)+\epsilon A \hat{X}(x) \exp (\omega t+\mathrm{i} \sigma y), \\
\boldsymbol{v}(x, y, t) & =\boldsymbol{v}_{b}(x ; \epsilon)+\epsilon A \hat{\boldsymbol{v}}(x) \exp (\omega t+\mathrm{i} \sigma y), \\
P(x, y, t) & =P_{b}(x ; \epsilon)+\epsilon A \hat{P}(x) \exp (\omega t+\mathrm{i} \sigma y),
\end{aligned}
$$

where $A \ll 1$ is the amplitude of the perturbed reaction sheet, $\sigma$ (real) is the wavenumber and $\omega$ (complex) is the growth rate. The auxiliary function $\delta$ is, in general, a function of $y$ and $t$ and, since it appears in the formulation only when expanding the $O(\epsilon)$ leakage terms, its proper perturbation is of the form

$$
\delta=\delta_{b}(\epsilon)+A \hat{\delta} \exp (\omega t+\mathrm{i} \sigma y)
$$

Substituting into the governing equations, boundary and jump conditions and retaining $O(\epsilon A)$ terms, leads to a linear complex eigenvalue problem for the perturbed quantities $\hat{T}, \hat{Y}, \hat{X}, \hat{u}, \hat{v}, \hat{P}$.

For simplicity of notation the operator $\mathrm{D} \equiv \mathrm{d} / \mathrm{d} x$ is introduced and the 'hat' accents are dropped. Introducing the vorticity perturbation

$$
\varpi=\mathrm{D} v-\mathrm{i} \sigma u
$$

and the stream-like function perturbation $\psi$ from the relations

$$
v=\mathrm{i} \sigma T-\mathrm{D} \psi, \quad u=\mathrm{i} \sigma \psi+\mathrm{D} T,
$$

then rescaling all variables according to

$$
\{\delta, T, Y, X, \psi, \varpi\} \Longrightarrow\left\{\sigma^{2} \delta, \sigma^{2} T, \sigma^{2} L_{F} Y, \sigma^{2} L_{X} X, \mathrm{i} \sigma \psi, \mathrm{i} \sigma \varpi\right\}
$$

yields the following eigenvalue problem:

$$
\begin{gathered}
\omega \rho_{b} T+M \mathrm{D} T+\rho_{b} \mathrm{D} T_{b}(\mathrm{D} T-M T-\psi)=\left(\mathrm{D}^{2}-\sigma^{2}\right) T \\
L_{F}\left(\omega \rho_{b} Y+M \mathrm{D} Y\right)+\rho_{b} \mathrm{D} Y_{b}(\mathrm{D} T-M T-\psi)=\left(\mathrm{D}^{2}-\sigma^{2}\right) Y \\
L_{X}\left(\omega \rho_{b} X+M \mathrm{D} X\right)+\rho_{b} \mathrm{D} X_{b}(\mathrm{D} T-M T-\psi)=\left(\mathrm{D}^{2}-\sigma^{2}\right) X \\
\omega \rho_{b} \varpi+M \mathrm{D} \varpi-\sigma^{2} M \rho_{b} \mathrm{D} T_{b}(\mathrm{D} T-M T-\psi)=\operatorname{Pr}\left(\mathrm{D}^{2}-\sigma^{2}\right) \varpi-\omega \mathrm{D} \rho_{b}\left(\sigma^{2} T-\mathrm{D} \psi\right) \\
\varpi=-\left(\mathrm{D}^{2}-\sigma^{2}\right) \psi
\end{gathered}
$$

with the boundary conditions

$$
\begin{gathered}
T=Y=X=\varpi=\psi-\mathrm{D} T=0 \quad \text { at } x=0, \\
T=Y=X=\varpi=\psi=0 \quad \text { as } x \rightarrow-\infty,
\end{gathered}
$$


and interfacial conditions, at the mean location of the perturbed reaction sheet $x=\eta_{0}$,

$$
\begin{gathered}
\llbracket T+Y \rrbracket=0, \quad \llbracket T+X \rrbracket=0, \\
M\left(L_{F}-1\right) \llbracket T \rrbracket+\llbracket \mathrm{D}(T+Y) \rrbracket=0, \\
M\left(L_{X}-1\right) \llbracket T \rrbracket+\llbracket \mathrm{D}(T+X) \rrbracket=0, \\
\llbracket \psi \rrbracket=\llbracket \mathrm{D} T-M T \rrbracket, \quad \llbracket \mathrm{D} \psi \rrbracket=\llbracket \sigma^{2} T \rrbracket, \\
\llbracket \varpi \rrbracket=\llbracket \mathrm{D} \varpi \rrbracket=0 \\
\left.Y\right|_{x=\eta_{0^{+}}}=\delta \frac{\partial S_{F}}{\partial \delta}\left(\gamma, \delta_{b}\right),\left.\quad X\right|_{x=\eta_{0^{-}}}=\delta \frac{\partial S_{X}}{\partial \delta}\left(\gamma, \delta_{b}\right),
\end{gathered}
$$

where

$$
\delta=\delta_{b}\left\{\left.\frac{1}{2}(1-\gamma)(T+Y)\right|_{x=\eta_{0^{+}}}+\left.\frac{1}{2}(1+\gamma)(T+X)\right|_{x=\eta_{0^{-}}}\right\} .
$$

This tenth-order homogeneous system possesses nontrivial solutions if and only if a solvability condition of the form

$$
\mathscr{F}\left(\omega, \sigma^{2} ; \mathbb{D}, L_{F}, L_{X}, \phi, r, \operatorname{Pr}, \Delta T\right)=0
$$

is satisfied, with $\omega=\omega_{R}+\mathrm{i} \omega_{I}$. For a given set of the parameters, $\mathbb{D}, L_{F}, L_{X}, \phi, r, \Delta T$ this functional dependence is a dispersion relation of the form $\omega=\omega\left(\sigma^{2}\right)$. If the real part $\omega_{R}<0$ for all wavenumbers $\sigma$, the planar flame is linearly stable; it is unstable if there exists at least one value of $\sigma$ for which $\omega_{R}>0$. The determination of the growth rate over the entire complex $\omega$ plane for a whole range of parameters is a formidable task that must be carried out numerically in a judicious way.

The numerical procedure employed for solving the eigenvalue problem (4.1)-(4.14) is similar to the one used by Metzener \& Matalon (2006) when solving the reduced system for the constant-density case. The first step is to transform through an appropriate change of variables each of the two domains into finite intervals of unit length, then divide the fuel region into $m+1$, and the oxidizer region into $n+1$ equidistant grid points. Appropriate discretization of the governing equations, boundary and interfacial conditions (see the referred paper) yields a generalized eigenvalue problem of the type

$$
\boldsymbol{A} \boldsymbol{x}=\omega \boldsymbol{B} \boldsymbol{x},
$$

where $\boldsymbol{A}$ and $\boldsymbol{B}$ are $N \times N$ real matrices, with $N=5(n+m+2)$ and the vector $\boldsymbol{x} \in \mathbb{C}^{N}$. The equations for $T, Y, X$ and $\varpi$ provide non-zero entries into $4(n+m)$ rows in both $\boldsymbol{A}$ and $\boldsymbol{B}$. Equation (4.5) for $\psi$ and the ten connection relations at the reaction sheet provide the additional $n+m+10$ rows with zero entries in the matrix $\boldsymbol{B}$. The matrix $\boldsymbol{B}$ is therefore non-invertible, requiring a non-standard numerical algorithm for the determination of the eigenvalues $\omega$. The f02bjf routine of the NAG library, which is applicable to eigenvalue problems similar to (4.15), was used to systematically follow the eigenvalues while varying the parameters. The procedure was employed successfully when analysing the linear stability problem for the constant-density model (Metzener \& Matalon 2006), and was shown to reproduce with high-accuracy results of the exact analytical dispersion relation (Cheatham \& Matalon 2000; Kukuck \& Matalon 2001).

Since the numerical procedure computes all the eigenvalues, it is necessary to find a compromise between the order of the matrices and the anticipated precision of the results. Convergence tests show that in most cases $n=m=25$ interior points are sufficient for characterizing the nature of the spectrum. Indeed, when the wavenumber $\sigma$ becomes large, $n$ and $m$ must be increased accordingly. 
Symbol Pattern

$\begin{array}{ll}\times & \text { Stationary cells } \\ + & \text { Planar pulsations } \\ \square & \text { Oscillatory cells } \\ \diamond & \text { High-frequency modes }\end{array}$

Reaction sheet of the marginal state

$x_{f}=\eta_{0}+\epsilon\left\{\eta_{1}+A \exp \left(\mathrm{i} \sigma^{*} y\right)\right\}$

$x_{f}=\eta_{0}+\epsilon\left\{\eta_{1}+A \exp \left(\mathrm{i} \omega_{I}^{*} t\right)\right\}$

$x_{f}=\eta_{0}+\epsilon\left\{\eta_{1}+A \exp \left[\mathrm{i}\left(\omega_{I}^{*} t \pm \sigma^{*} y\right)\right]\right\}$

$x_{f}=\eta_{0}+\epsilon\left\{\eta_{1}+A \exp \left[\mathrm{i}\left(\tilde{\omega}_{I}^{*} t / \epsilon^{2} \pm \tilde{\sigma}^{*} y / \epsilon\right)\right]\right\}$

TABLE 1. Possible patterns of flame front instability and the corresponding symbols used to identify these modes in the stability diagrams.

\section{Stability results}

For linear stability considerations one needs to focus on the eigenvalue with the largest real part, which will be denoted here by $\omega_{R}$. Of interest is the behaviour of $\omega_{R}$ as one of the parameters varies, retaining all the others fixed. An instability results when $\omega_{R}$ crosses the real axis and the nature of the instability is characterized by the wavenumber $\sigma^{*}$ and frequency $\omega_{I}^{*}$ of the neutral state. When $\omega_{I}^{*}=0$ stationary cells with characteristic size $\sim 2 \pi / \sigma^{*}$ result at the instability threshold. When $\omega_{I}^{*} \neq 0$, planar pulsations result if $\sigma^{*}=0$ and oscillating cells with dimensions $\sim 2 \pi / \sigma^{*}$ otherwise; in either case the characteristic frequency is proportional to $\omega_{I}^{*}$. High-frequency modes characterized by $\omega_{I} \gg 1$ and/or $\sigma \gg 1$ result in extreme conditions; e.g. states that are very close to extinction. A more precise characterization of these modes requires incorporating in the analysis disturbances of wavelength comparable to the reaction zone thickness, that evolve on the fast reaction time. Instabilities then arise with $O\left(\epsilon^{2} \omega^{*}\right)$ frequencies and $O\left(\epsilon \sigma^{*}\right)$ wavenumbers. Further details, including citation of previous studies on these high-frequency modes can be found in Metzener \& Matalon (2006). In the stability diagrams presented below we have used different symbols to identify the different modes of instability; the symbols and their respective definitions are summarized in table 1.

In the calculations presented below we have selected $P r=0.5$, which is a typical value for common combustion mixtures and, for simplicity, taken $\Delta T=0$. Then, given $L_{F}, L_{X}, \phi, r$, the basic state is parametrized by $\mathbb{D}$. Starting with a stable solution for which all the eigenvalues are on the left half of the complex plane, the motion of the eigenvalues is tracked by systematically varying $\mathbb{D}$ until an eigenvalue crosses the imaginary axis. The marginal state is denoted by $\mathbb{D}^{*}$. The calculations are repeated by spanning a range of all relevant parameters.

\subsection{The Burke-Schumann flame sheet}

A straightforward conclusion from the exact analytical dispersion relation of the constant-density model is that the Burke-Schumann limit of complete combustion $(\mathbb{D} \rightarrow \infty)$ is unconditionally stable. This conclusion is re-examined below for a variable-density flow. In figure 4 the dependence of the growth rate $\omega_{R}$ on $\sigma$ is shown for an increasing range of Damköhler number. The two illustrations show that an instability develops only when $\mathbb{D}$ is sufficiently small. The parameters were chosen such that the unstable state beyond the instability threshold corresponds either to stationary cells or planar pulsations, but the results have been tested for a wider range of parameter values. Figure $4(a)$ is obtained for $L_{F}=L_{X}=0.7$ and $\phi=1.0$ with $M=3.33$ corresponding to a density ratio $r=3.095$ across the flame. A neutral state exists for $\mathbb{D}^{*} \approx 4.795$ with a critical wavenumber $\sigma^{*} \approx 3$ and $\omega_{I}=0$. The instability just beyond the marginal state is, therefore, associated with the evolution of stationary cells. Figure $4(b)$ is obtained for $L_{F}=L_{X}=1.25$ and $\phi=1.25$ with $M=4$ 
(a)

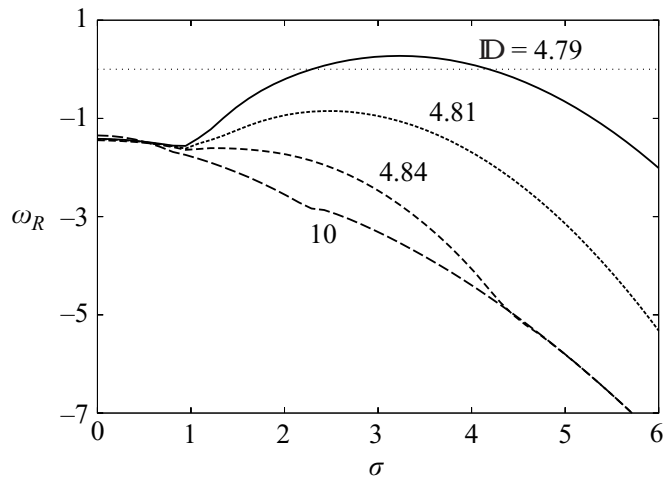

(b)

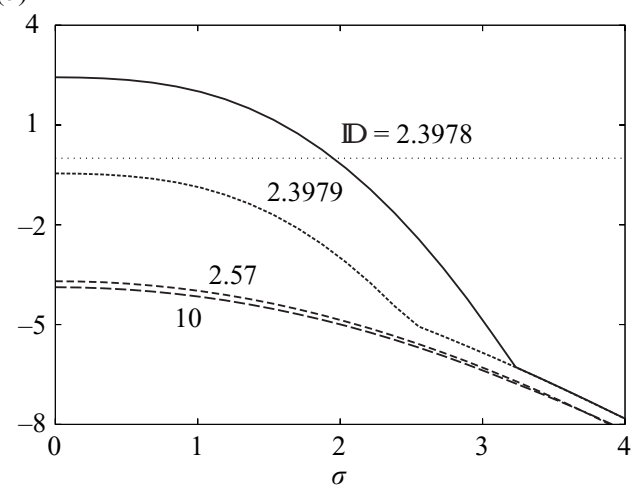

FIgURE 4. The growth rate $\omega_{R}$ as a function of the wavenumber $\sigma$ for increasing Damkhöler numbers $\mathbb{D}$. The parameters in these two illustrations were chosen for conditions that lead beyond the instability threshold to $(a)$ stationary cells and $(b)$ planar pulsations. The figures show that for large $\mathbb{D}$ the flame is always stable.

corresponding to a density ratio $r=2.5$ across the flame. A neutral state exists for $\mathbb{D}^{*} \approx 2.39788$ with $\omega_{I} \neq 0$ but with the critical wavenumber $\sigma^{*}=0$. The instability that evolves beyond the marginal state is, therefore, associated with planar pulsations. In both cases, it is evident that $\omega_{R}<0$ when $\mathbb{D} \rightarrow \infty$ for all $\sigma$, implying that the Burke-Schumann flame sheet is unconditionally stable. When a planar flame is slightly perturbed, the requirement of complete reactant consumption, $Y=X=0$, forces the reaction sheet to assume its original planar form which, for the given parameters, is the only stoichiometric surface. Instabilities can only appear when $\mathbb{D}<\mathbb{D}^{*}$ or where there is appreciable reactant leakage through the reaction sheet (see figure 3).

The graphs in figure 4 show that the largest eigenvalue $\omega_{R}$ has two different origins, which identifies the existence of two separate modes. A purely diffusive mode which is always stable and is the only one that survives as $\mathbb{D} \rightarrow \infty$, and a reactive-diffusive mode which has its origin in the reaction and diffusion processes occurring in the thin reaction zone and is therefore very sensitive to variations in the Damköhler number.

\subsection{Equi-diffusion flames}

The exact dispersion relation for the constant-density case simplifies greatly when the Lewis numbers are both identically equal to one, from which it may be easily concluded that the planar flame is unconditionally stable. While this conclusion remains true even when density variations are accounted for, it is of little practical interest because $L_{F}=L_{X}=1$ is a condition that cannot be met in practice. Unequal diffusivities however small have the tendency to destabilize the flame when $\mathbb{D}$ is sufficiently low as will become clear from the stability diagram of figure 8 discussed later in this paper.

\subsection{Thermal expansion}

Assuming that both reactants are supplied at the same temperature $(\Delta T=0)$, the effect of thermal expansion is characterized by the density ratio $r$. As before, we illustrate the results for parameters associated with the onset of stationary cellular structures and planar pulsations. Given the parameters $L_{F}, L_{X}, \phi$ and $r$ the basic state is parameterized by the Damköhler number $\mathbb{D}$. Since fixing $\mathbb{D}$ and computing the corresponding $\delta$ for the determination of the reactant leakage or using the auxiliary parameter $\delta$ is equivalent, we choose for convenience to identify the various states by varying $\delta$ directly. Figure 5 corresponds to $L_{F}=L_{X}=0.75$ and $\phi=0.5$ and illustrates 
(a)

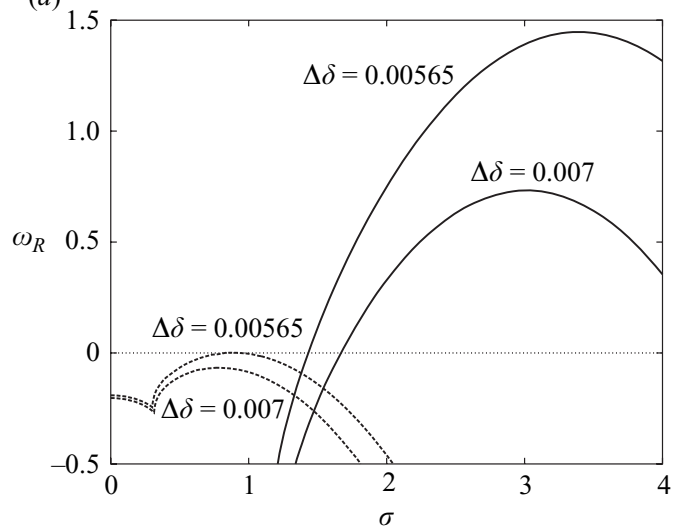

(b)

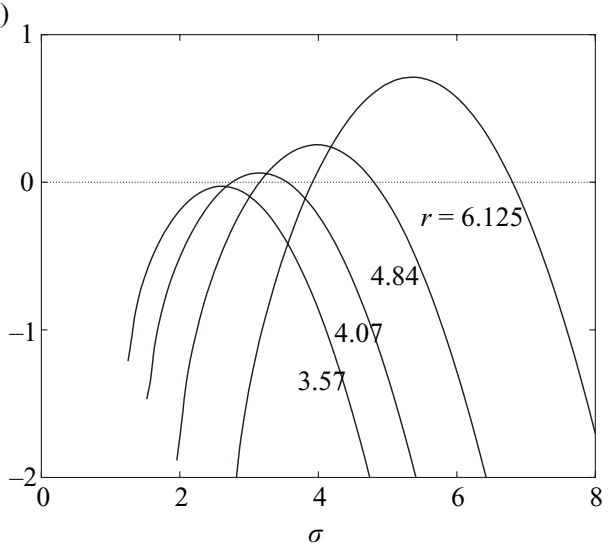

FIGURE 5. The effect of thermal expansion on the onset of the cellular instability. ( $a$ ) Comparison of the growth rate of the constant- (dashed curves) and variable-density (solid curves) models for two values of the Damköhler number. ( $b$ ) Effect of density variations $r$ on the growth rate for a fixed value of the Damköhler number.

the effect of thermal expansion on the onset of the cellular instability. In figure $5(a)$ we show a comparison of the growth rate $\omega_{R}$ for two values of the Damköhler number, or $\Delta \delta \equiv \delta-\delta_{c}$, for the constant- (dashed curves) and variable-density (solid curves) models. For the variable-density model we have chosen $T_{-\infty}=0.3$, which corresponds to a density ratio $r=3.56$. Based on the constant-density model the planar flame is stable for $\Delta \delta=0.007$ and marginally stable for a slightly smaller value $\Delta \delta \approx 0.00565$. Based on the variable-density model the planar flame is unstable for both values of $\Delta \delta$; the onset of instability occurred at a much larger value of the Damköhler number. In both cases the imaginary part corresponding to $\omega_{R}$ at criticality vanishes implying that the bifurcation is to a steady structure. Since the neutrally stable state is attained for a finite value $\sigma=\sigma^{\star}$, the state beyond the instability threshold is a cellular structure with dimensions $\sim 2 \pi / \sigma^{\star}$. Thermal expansion has, therefore, a destabilizing influence on the onset of cellular flames and, as a result, a wider range of physical states than predicted by the constant-density model is susceptible to the cellular instability. Moreover, the wavenumber of the fastest growing mode at criticality increases with increasing $r$, implying that the constant-density model slightly overestimates the cell dimension. In figure $5(b)$ we show the dependence of the growth rate $\omega_{R}$ at a fixed value of $\mathbb{D}$ (corresponding to $\Delta \delta \approx 0.0092$ ) for several increasing values of $r$. The degree of instability increases with increasing $r$ and the band of growing modes is larger.

While the origin of the cellular instability lies in the competing effects of thermal and mass diffusion requiring, in general, subunity Lewis numbers, the effect of thermal expansion is associated with the flow induced by the dramatic change in density near the reaction sheet. The flow field near the corrugated sheet involves regions of concentrated vorticity with extrema near the crests and troughs (see figure 6). Since a diffusion flame does not possess an intrinsic propagation speed and the reaction sheet lies where the reactant concentrations and their fluxes satisfy conditions (2.11)-(2.12), the induced vortical motion enhances the transport of reactants towards the crests and troughs, which sustains the cellular structure.

Figure 7 corresponds to $L_{F}=L_{X}=1.25$ and $\phi=1.25$ and illustrates the effect of thermal expansion on the pulsating instability. In figure $7(a)$ we show the growth rate $\omega_{R}$ for two values of the Damköhler number, or $\Delta \delta$, for the constant- (dashed 


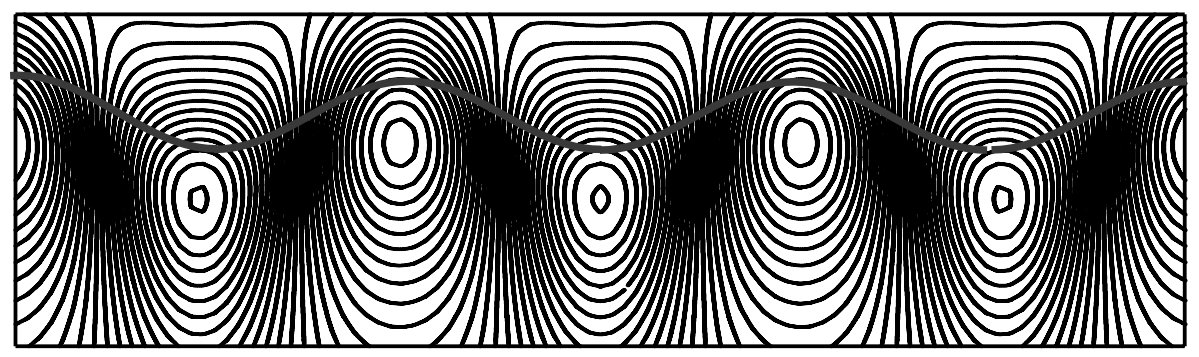

FIGURE 6. The vorticity field in the vicinity of the corrugated reaction sheet surface (solid thick curve) for conditions near the threshold of the cellular instability.

curves) and variable-density (solid curves) models. For the variable-density model we have chosen $T_{-\infty}=0.25$, which corresponds to a density ratio $r=2.5$. Based on the constant-density model the planar flame is unstable for both values of $\Delta \delta$. Based on the variable-density model the flame is unstable for $\Delta \delta=0.01127$ and stable for the smaller $\Delta \delta=0.0113$, with marginal stability occurring at an intermediate value. In both cases the imaginary part corresponding to $\omega_{R}$ does not vanish at criticality implying that the bifurcation is to an oscillatory state with frequency $\sim \omega_{I}^{\star}$. Since the most dangerous mode at the instability threshold is $\sigma=0$, the flame remains planar but oscillates back and forth normal to itself. Thermal expansion has, therefore, a stabilizing influence on the onset of planar pulsations and as a result a narrower range of physical states than predicted by the constant-density model is susceptible to this instability. In figure $7(b)$ we show the dependence of the growth rate $\omega_{R}$ at a fixed value of $\mathbb{D}$, for several increasing values of $r$. The graph shows the stabilizing influence of thermal expansion with the degree of instability decreasing with increasing $r$.

While the origin of the pulsating instability lies in the competing effects of thermal and mass diffusion requiring, in general, sufficiently large Lewis numbers, the effect of thermal expansion is associated with the flow induced by the rapid change in density near the reaction sheet. Since the temperature peaks at the reaction sheet, during its back and forth motion the flame move towards a denser gas which has a damping effect on its motion.

\subsection{Differential and preferential diffusion}

We now examine the effect of non-equal and non-unity Lewis numbers for stoichiometric conditions $\phi=1$. The representative value $T_{-\infty}=0.3$, which corresponds to a density ratio $r=3.56$, was chosen for these calculations. The results are displayed in the Lewis numbers parameter plane shown in figure 8 , which covers values relevant to most combustible mixtures of interest. The diagram, which summarizes results of a large number of stability computations, identifies for a given set of Lewis numbers $L_{F}$ and $L_{X}$ the nature of the instability and the pattern that evolves when the Damkhöler number is reduced below criticality, i.e. just below $\mathbb{D}^{*}$. The Lewis numbers may be individually controlled by appropriately diluting the fuel and/or the oxidizer supply with inert gas. The different symbols in this diagram correspond to the patterns identified in table 1 . The solid curve corresponds to $h_{f}=0$ and separates regions of excess (below the curve) and deficiency (above the curve) in the available enthalpy at the reaction sheet. One observes, in general, a distinction between the instability patterns when both Lewis numbers are less/greater than one. For $L_{F}$, $L_{X}<1$ a stationary two-dimensional cellular structure is supported by the ability 
(a)

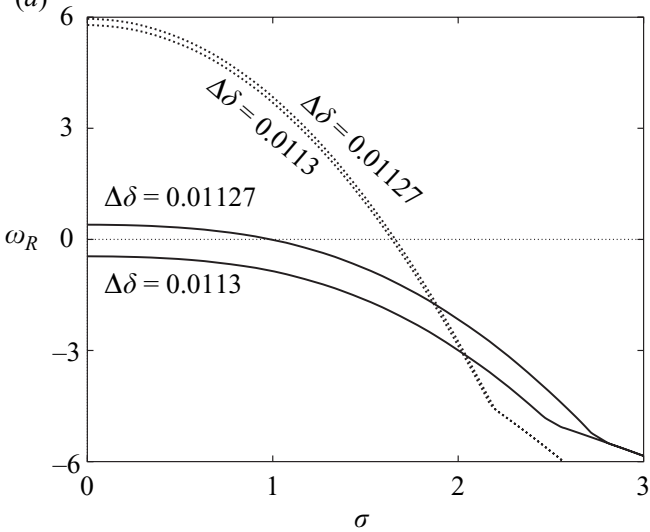

(b) 10

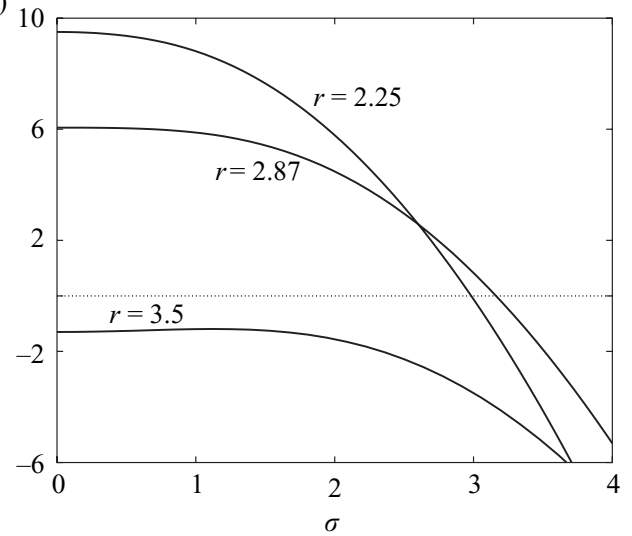

FiguRE 7. The effect of thermal expansion on the onset of planar pulsations. (a) Comparison of the growth rate for constant- (dashed curves) and variable-density (solid curves) for two different values of the Damköhler number. $(b)$ Effect of density variations $r$ on the growth rate for a fixed Damköhler number.

of the reactants to diffuse towards the reaction zone faster than removal of heat by conduction (excess in the available enthalpy). For $L_{F}, L_{X}>1$ heat is conducted from the reaction zone at a faster rate than the reactants are able to diffuse towards the sheet (deficiency in the available enthalpy). The reaction sheet locates itself where conditions are more favourable and thus moves back and forth relative to the stoichiometric surface. In this region the flame retains its planar shape and undergoes planar pulsations. This mode of instability is also the preferred mode when $L_{X}<1$ and $L_{F}>1$, except that now the frequency of oscillations increases significantly resulting in what has been referred to as high-frequency modes (Metzener \& Matalon 2006). When $L_{F}<1$ and $L_{X}$ is sufficiently large, the instability is in the form of oscillating cells, a combination of the competing modes. In the present configuration fuel is supplied in the stream and is thus more readily available in the reaction zone than oxidizer that must diffuse against the stream, which explains the asymmetry in the predicted patterns seen in the upper-left and lower-right corners of the diagram.

Additional details are shown in figure 9, restricted to $(a)$ changes that occur when $L_{X}$ is increased continuously while retaining $L_{F}=0.7$ fixed, i.e. as one moves along a horizontal line of the stability diagram, and $(b)$ changes that occur when $L_{F}$ is increased continuously while retaining $L_{X}=1.5$ fixed, i.e. as one moves along a vertical line of the stability diagram. Plotted in the figures are: the marginal Damköhler number, presented by its value relative to the extinction state $\Delta \mathbb{D}^{*}=\left(\mathbb{D}^{*}-\mathbb{D}_{\text {ext }}\right) / \mathbb{D}_{\text {ext }}$, the critical wavenumber $\sigma^{*}$ at the onset of instability, and the critical frequency $\omega_{I}^{*}$. Starting at a low value of $L_{X}$ in figure $9(a)$, the bifurcation that occurs at $\mathbb{D}^{*}$ is to a steady cellular structure with dimension $\sim 2 \pi / \sigma^{*}$. The range of unstable states, measured relative to the extinction state, is reduced when $L_{x}$ increases and the predicted cell size gets smaller. When the onset of instability nearly coincides with extinction $\left(\Delta \mathbb{D}^{*} \approx 0\right)$ the resulting cell size is extremely small and comparable to the reaction zone thickness, conditions referred to as high-frequency modes. This mode of instability, often referred to as the fast time instability, is relevant to limited conditions that are seldom observed in practice; experimentally observed cellular structures are typically of dimensions comparable to the diffusion length (Robert 2008). When $1.25<L_{x}<1.5$ the flame remains planar $\left(\sigma^{*}=0\right)$ but oscillates 


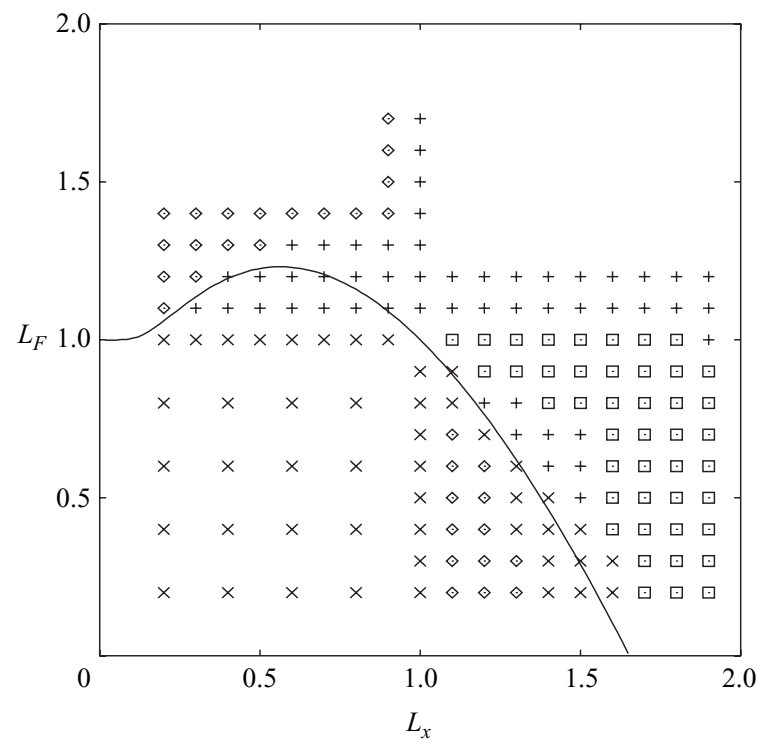

FIGURE 8 . Stability diagram illustrating the nature of the instability for different values of the Lewis numbers; the definitions of the different symbols are listed in table 1.

(a)
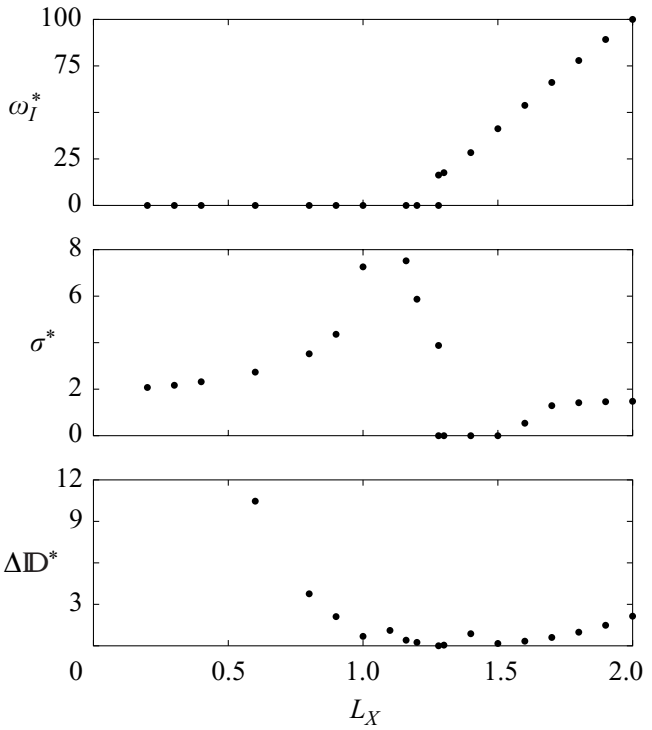

(b)
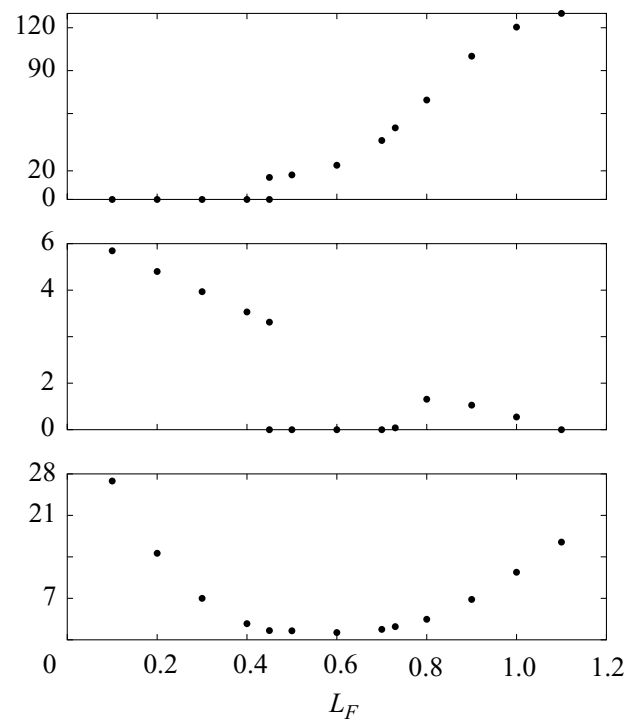

FIGURE 9. Dependence of marginal state $\Delta \mathbb{D}^{*}$, critical wavenumber $\sigma^{*}$ and frequency $\omega_{I}^{*}$ on $(a)$ $L_{X}$ for fixed $L_{F}=0.7$ and $(b)$ for $L_{F}$ for fixed $L_{X}=1.5$. The results in both figures correspond to $\phi=1$ and $r=3.56$.

at a frequency $\sim \omega_{I}^{*}$ that increases with increasing $L_{X}$. Beyond $L_{X}=1.5$, the instability is in the form of oscillating cells. The critical wavenumber $\sigma^{*}$ increases with increasing $L_{X}$ and reaches a constant value as $L_{X}$ becomes sufficiently large, nearly half its value for small values of $L_{x}$. The oscillating cells are typically of large dimensions, much larger than the stationary cells observed at low Lewis numbers. Starting at a low value of $L_{F}$ in figure $9(b)$, the bifurcation that occurs at a sufficiently large value of $\mathbb{D}$ 
is to a steady cellular structure. Planar pulsations is the preferred mode of instability when $L_{F}>0.4$, with oscillating cells of large dimensions emerging for $0.8<L_{F}<1$. The passage from stationary cells to planar pulsations in this case does not evolve through transitional high-frequency modes; rather the two patterns are marginally stable at $L_{F}=0.45$. The various instability modes shown in figure 8 are, therefore, not separated by sharp boundaries and the passage from one mode to another occurs through competing modes of instability of comparable or disparate scales.

Finally, we note that a comparison of figure 8 with the equivalent diagram presented by Metzener \& Matalon (2006) shows that the general characterization of the instabilities predicted by the constant-density model remain qualitatively correct with the differences primarily in the transitional regions and in the conditions characterizing the onset of instability (see the earlier discussion about the effect of thermal expansion).

\subsection{Mixture strength}

The location where $\gamma=0$ separates regions in the stability diagram of figure 8 where the available mixture in the reaction zone is relatively lean (to the left) or rich (to the right). For $\phi=1$ this location coincides with the vertical line $L_{X}=1$ (not shown in the figure) as evident from (3.8); it moves to the right when reducing $\phi$ and to the left when increasing $\phi$. The overall effect of varying the mixture strength $\phi$ on stability is similar to the results presented for the constant-density model. The region where stationary cells are predicted stretches out when reducing $\phi$ to include a wider range of $L_{X}$, implying that leaner conditions are more susceptible to cellularity. On the other hand, since the location of $\gamma=0$ moves to the left as $\phi$ increases, oscillations are favoured for rich conditions.

\section{Conclusions}

In this paper we have examined the effect of thermal expansion on the stability of diffusion flames. Although thermal expansion does not play a dominant role as it does for premixed flames, it nevertheless has a significant effect on the critical conditions for the onset of instabilities and on the degree of instability. When the reaction is infinitely fast (the Burke-Schumann limit of complete combustion) the flame is unconditionally stable. Instabilities arise only when combustion is incomplete and significant amount of fuel and/or oxidizer leak through the reaction zone, i.e. for Damköhler numbers in the range $\mathbb{D}_{\text {ext }} \leqslant \mathbb{D}<\mathbb{D}^{*}$. Since the characteristic flow time for the chambered flame is $l_{D} / U$, where $l_{D}=\mathscr{D}_{t h} / U$ is the diffusion length and $U$ is the bulk flow velocity, this corresponds to relatively high flow rates or near-extinction conditions consistent with experimental observations. The driving mechanism for instabilities is the competing effects of heat conduction away and mass diffusion towards the reaction zone, with the predicted dynamical behaviours depending primarily on differential and preferential diffusion, flow rates, supply conditions and mixture strength. Thermal expansion was found to play a secondary role, and has different effects on the various modes of instability. In particular, it has a destabilizing influence on the formation of cells and a stabilizing influence on the onset of planar pulsations. The onset of cellular flame occurs at a larger value $\mathbb{D}^{*}$ than that predicted by the constant-density model, so that a wider range of states $\mathbb{D}^{*}-\mathbb{D}_{\text {ext }}$ are susceptible to the cellular instability. The onset of planar pulsations, on the other had, occurs at a smaller value $\mathbb{D}^{*}$ than that predicted by the constant-density model, so that a narrower range of states $\mathbb{D}^{*}-\mathbb{D}_{\text {ext }}$ are susceptible to pulsations. Because of the rich dynamical behaviour the emerging pattern in transitional states between the various modes of instability may be quite complex. The influence of thermal expansion in these circumstances will depend 
on the competing instability modes. Finally, we point out that heating one of the reactants changes the temperature differential and consequently the density contrast across the entire combustion layer; its influence may either combine or compete with the effect of thermal expansion.

This work was partially supported by the National Science Foundation under grant DMS-0708588.

\section{REFERENCES}

Burke, S. P. \& Schumann, T. E. W. 1928 Diffusion flames. Ind. Engng Chem. 20, 998.

ChAN, W. \& Tien, J. S. 1979 An experiment on spontaneous flame oscillation prior to extinction. Combust. Sci. Technol. 18, 139-143.

Cheatham, S. \& Matalon, M. 2000 A general asymptotic theory of diffusion flames with application to cellular instability. J. Fluid Mech. 414, 105-144.

Chen, R., Mitchell, G. B. \& Ronney, P. D. 1992 Diffusive-thermal instability and flame extinction in nonpremixed combustion. Proc. Combust. Inst. 24, 213-221.

Darrieus, G. 1938 Propagation d'un front de flamme. Presented at La Technique Moderne, Paris.

Dongworth, M. R. \& Melvin, A. 1976 The transition to instability in a steady hydrogen-oxygen diffusion flame. Combust. Sci. Technol. 14, 177-182.

FÜrI, M., PAPAs, P. \& Monkewitz, P. A. 2000 Nonpremixed jet flame pulsations near extinction. Proc. Combust. Inst. 28, 831-838.

Gardside, J. E. \& JaCKSON, B. 1951 Polyhedral diffusion flames. Nature 168, 1085-1085.

Golovanevsky, B., Levy, Y., Greenberg, J. B. \& Matalon, M. 1999 On the oscillatory behaviour of laminar spray diffusion flames: experiment and theory. Combust. Flame 117, 373-383.

IshizukA, S. \& TsujI, H. 1981 An experimental study of effect of inert gases on extinction of laminar diffusion flames. Proc. Combust. Inst. 18, 695-703.

Kim, J. S., Williams, F. A. \& Ronney, P. D. 1996 Diffusional-thermal instability of diffusion flames. J. Fluid Mech. 327, 273-301.

Kunuck, S. \& Matalon, M. 2001 The onset of oscillations in diffusion flames. Combust. Theory Modelling 5, 217-240.

Landau, L. D. 1944 On the theory of slow combustion. Acta Physicochim. USSR 19, 77-85.

LIÑÁN, A. 1974 The asymptotic structure of counterflow diffusion flames for large activation energies. Acta Astronaut. 2, 1009.

Lo JACOnO, D. \& Monkewitz, P. A. 2007 Scaling of cell size in cellular instabilities of non-premixed jet flames. Combust. Flame. 151 (1-2), 321-332.

Lo Jacono, D., Papas, P., Matalon, M. \& Monkewitz, P. A. 2005 An experimental realization of an unstrained planar diffusion flame. Proc. Combust. Inst. 30, 501-509.

Lo Jacono, D., Papas, P. \& Monkewitz, P. A. 2003 On the formation of cellular instabilities in non-premixed jet flames. Combust. Theory Modelling 7, 634-644.

Metzener, P. \& Matalon, M. 2006 Diffusive-thermal instabilities of diffusion flames: onset of cells and oscillations. Combust. Theory Modelling 10 (4), 701-725.

Miklavcic, M., Moore, A. B. \& Wichman, I. S. 2005 Oscillations and island evolution in radiating diffusion fames. Combust. Theory Modelling 9, 403-416.

RoBert, E. 2008 Experimental investigation of unstrained diffusion flames and their instabilities. $\mathrm{PhD}$ thesis, Ecole Polytechnique Federale de Lausanne, Lausanne.

Robert, E. \& Monkewitz, P. A. 2009 Experiments in a novel quasi-1d diffusion flame with variable bulk flow. Proc. Combust. Inst. 32, 987-994.

Ross, H., Sotos, R. G. \& T'IEN, J. S. 1991 Observations of candle flames under various atmospheres in microgravity. Combust. Sci. Technol. 75, 155-160.

Ross, H. D. 1994 Ignition of and flame spread over laboratory-scale pools of pure liquid fuels. Prog. Energy Combust. Sci. 20 (1), 17-64.

Schiller, D. N., Ross, H. D. \& Sirignano, W. A. 1996 Computational analysis of flame spread across alcohol pools. Combust. Sci. Technol. 118, 203-255.

Vance, R., Miklavcic, M. \& Wichman, I. S. 2001 On stability of one-dimensional diffusion fames. Combust. Theory Modelling 5, 147-162. 